\title{
Hybrid Statistical Model Checking Technique for Reliable Safety Critical Systems
}

\author{
Youngjoo Kim, Moonzoo Kim \\ Computer Science Department, KAIST \\ South Korea \\ jerry88@cs.kaist.ac.kr, moonzoo@cs.kaist.ac.kr
}

\begin{abstract}
Reliability of safety critical systems such as nuclear power plants and automobiles has become a significant issue to our society. As more computing systems are utilized in these safety critical systems, there are high demands for verification and validation $(\mathrm{V} \& \mathrm{~V})$ techniques to assure the reliability of such complex computing systems. However, as the complexity of computing systems increases, conventional $\mathrm{V} \& \mathrm{~V}$ techniques such as testing and model checking have limitations, since such systems often control highly complex continuous dynamics. To improve the reliability of such systems, statistical model checking (SMC) techniques have been proposed. SMC techniques can check if a target system satisfies given requirements through statistical methods. In this paper, we propose a new hybrid SMC technique that integrates sequential probability ratio test (SPRT) technique and Bayesian interval estimation testing (BIET) technique to achieve precise verification results quickly. In our experiment, the new hybrid SMC was up to $20 \%$ faster than BIET. In addition, we demonstrate the effectiveness and efficiency of this hybrid SMC technique by applying the hybrid SMC technique to three safety critical systems in the automobile domain.
\end{abstract}

\section{INTRODUCTION}

Various areas of our life utilize computing systems such as smart phones, medical devices, and automobile controllers. Consequently, the reliability of computing systems becomes a significant issue to our society and various international standards have been proposed and applied to assure reliability of such systems. For example, automobile domain has a functional safety standard ISO 26262 [7].

However, as computing power increases, the complexity of computing systems also increases rapidly, which causes many challenges to assure the reliability of computing systems. In particular, the size and complexity of software in a computing system has increased quickly. Although software reliability has been studied actively [14], conventional verification and validation $(\mathrm{V} \& \mathrm{~V})$ techniques for software such as testing and model checking [3] have limitations to assure the reliability of complex safety critical computing systems. One reason for this difficulty is that such systems often control highly complex continuous dynamics to interact with physical environments. In addition, since safety critical systems consist of both hardware and software and interact with a physical environment that often behaves non-deterministically (e.g., condition of road surface for automobiles or wind speed for airplanes), we should analyze target hardware and software with its environment together as a stochastic process [15]. However, conventional $\mathrm{V} \& \mathrm{~V}$ techniques for software have difficulty analyzing target systems in such contexts.
To improve the reliability of safety critical systems, statistical model checking (SMC) techniques [21], [19], [20], [6], [23], [4], [8] have been proposed. SMC techniques approximately compute probabilities for a target system to satisfy given requirements based on randomly sampled execution traces. Thus, SMC techniques can assure the reliability of a complex target system statistically without analyzing the internal logic of a target system.

In our previous work [9], we empirically evaluated the effectiveness (i.e., precision of verification) and efficiency (i.e., time cost of verification) of the four state-of-the-art SMC techniques including single sampling plan (SSP) [19], sequential probability ratio test (SPRT) [21], Bayesian hypothesis testing (BHT) [8], and Bayesian interval estimation testing (BIET) [23]. Through the empirical study, we observed that these SMC techniques have different strong points and weak points which may complement one another. From this observation, we developed a new hybrid SMC technique which combines SPRT, the fastest SMC technique, and BIET, the most precise SMC technique. This hybrid SMC technique achieves precise verification result fast. Although precise verification result is a top priority for safety critical systems, the time cost of verification cannot be ignored in practice. Thus, we can improve the reliability of safety critical systems more practically by applying our new hybrid SMC technique. To demonstrate the effectiveness and efficiency of this hybrid SMC technique, we have applied this hybrid SMC technique to three safety critical systems in the automobile domain - an automatic transmission control system (ATCS), an anti-lock braking system (ABS), and a fault-tolerant fuel control system (FFCS). Through the experiments, we confirmed that our hybrid SMC technique improves effectiveness and efficiency compared to a single SMC technique.

Section II overviews related SMC techniques. Section III describes a new hybrid SMC algorithm. Section IV explains the three target systems: ATCS, ABS, and FFCS. Section V describes the SMC results by using single SMC techniques and the hybrid technique on ATCS, ABS, and FFCS. Section VI discusses issues from the empirical study. Section VII concludes this paper with future work.

\section{OVERVIEW OF SMC TECHNIQUES}

\section{A. SMC Framework}

Figure 1 illustrates a SMC framework. There are two classes of SMC techniques: hypothesis testing and estimation testing. 


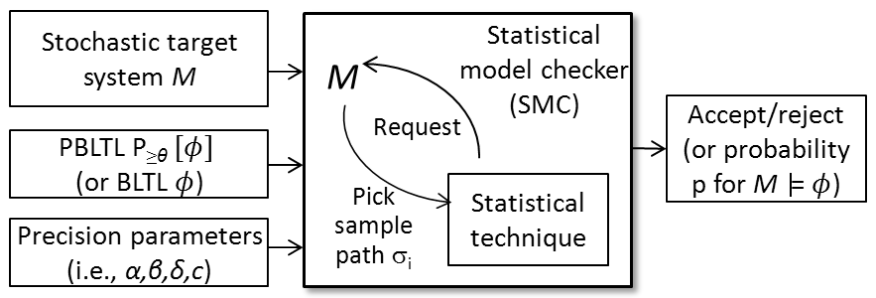

Fig. 1. Framework of SMC techniques

A hypothesis testing technique receives a target system $\mathcal{M}$, a probabilistic bounded linear temporal logic (PBLTL) [23] formula $P_{\geq \theta}[\phi]$ with probability threshold $\theta$, and precision parameters. A hypothesis testing technique produces an 'accept' answer if $\mathcal{M} \models P_{\geq \theta}[\phi]$ which means that a probability for $\mathcal{M}$ to satisfy $\phi$ is greater than or equal to $\theta$; a 'reject' answer, otherwise. An estimation testing technique receives a target system $\mathcal{M}$ and a bounded linear temporal logic (BLTL) [22] formula $\phi$ with precision parameters and produces an estimated probability $p$ regarding $\mathcal{M} \models \phi$.

To produce an answer, both classes of SMC techniques pick a random sample path $\sigma_{i}$ by executing $\mathcal{M}$ and collect the result of checking $\sigma_{i} \models \phi$. SMC techniques request a sample path repeatedly until the information of sample paths are enough to determine if $\mathcal{M}=P_{\geq \theta}[\phi]$ or to calculate $p$ for $\mathcal{M}=\phi$ with given precision parameters. Note that SMC techniques should determine a number of sample paths $n$ to check if $\mathcal{M} \models P_{\geq \theta}[\phi]$ or to calculate $p$ for $\mathcal{M} \models \phi$ using statistical techniques. Most SMC techniques calculate $n$ dynamically through iterative sampling.

\section{B. Probabilistic Bounded Linear Temporal Logic}

We define a syntax and semantics of bounded linear temporal logic (BLTL) [22] and PBLTL [23]. For a target model $\mathcal{M}, S V$ is a finite set of real-valued state variables. A Boolean predicate over $S V$ is a constraint of the form $y \sim v$, where $y \in S V, \sim \in\{\geq, \leq,=\}$, and $v \in \mathbb{R}$. The syntax of the BLTL logic formula $\phi$ is given by the following grammar:

$$
\phi::=y \sim v\left|\left(\phi_{1} \vee \phi_{2}\right)\right|\left(\phi_{1} \wedge \phi_{2}\right)\left|\neg \phi_{1}\right|\left(\phi_{1} \mathbf{U}^{t} \phi_{2}\right),
$$

where $y \in S V, \sim \in\{\geq, \leq,=\}, v \in \mathbb{R}$, and $t \in \mathbb{R}_{\geq 0}$.

For other temporal operators, we can define $\mathbf{F}^{t} \phi$ as True $\mathbf{U}^{t} \phi$ and $\mathbf{G}^{t} \phi$ as $\neg \mathbf{F}^{t} \neg \phi$. We denote a fact that an execution $\sigma$ satisfies a property $\phi$ as $\sigma \models \phi$. We use $\sigma^{k}$ to denote a suffix trace of $\sigma$ starting at step $k\left(\sigma^{0}\right.$ denotes the original execution $\sigma$ ). We denote the value of a state variable $y$ in $\sigma$ at step $k$ by $V(\sigma, k, y)$. We define $t_{k}$ as a time at step $k$ and $t$ as a time bound. The semantics of BLTL on a trace $\sigma^{k}$ is defined as follows:

- $\sigma^{k} \models y \sim v$ iff $V(\sigma, k, y) \sim v$

- $\sigma^{k} \models \phi_{1} \vee \phi_{2}$ iff $\sigma^{k}=\phi_{1}$ or $\sigma^{k} \models \phi_{2}$

- $\sigma^{k} \models \phi_{1} \wedge \phi_{2}$ iff $\sigma^{k} \models \phi_{1}$ and $\sigma^{k} \models \phi_{2}$

- $\sigma^{k} \models \neg \phi_{1}$ iff $\sigma^{k} \not \models \phi_{1}$

- $\sigma^{k} \models \phi_{1} \mathbf{U}^{t} \phi_{2}$ iff there exists $i \in \mathbb{N}$ such that

1) $\sum_{0 \leq l<i} t_{k+l} \leq t$,

2) $\sigma^{k+i} \models \phi_{2}$, and
3) for each $0 \leq j<i, \sigma^{k+j}=\phi_{1}$

A probabilistic bounded linear temporal logic (PBLTL) formula is a formula of the form $P_{\geq \theta}[\phi]$, where $\phi$ is a BLTL formula and $\theta \in(0,1)$ is a probability threshold. We denote that a model $\mathcal{M}$ satisfies PBLTL property $P_{\geq \theta}[\phi]$ as $\mathcal{M} \models P_{\geq \theta}[\phi]$, which means that a probability for $\mathcal{M}$ to satisfy $\phi$ is greater than or equal to $\theta$ (see [23] for detailed description).

\section{Sequential Probability Ratio Test}

Sequential probability ratio test (SPRT) is a hypothesis testing technique introduced by Younes et al. [21]. SPRT [21], [19], [20], [16] determines a number of required sample paths dynamically at runtime. The main goal of SPRT is to decide if $\mathcal{M} \models P_{\geq \theta}[\phi]$ with a small number of sample paths. If another sample path is needed, SPRT generates one more sample path by executing a target system. If the information from generated sample paths is enough, SPRT stops executing the target program and produces an answer regarding $\mathcal{M} \models P_{\geq \theta}[\phi]$. SPRT uses precision parameter inputs error bounds $\alpha$ and $\beta$, and a half size of indifference region $\delta$. The detailed description of SPRT is as follows.

Before building a hypothesis for hypothesis testing of SPRT, we introduce the indifference region. Basically, we build a hypothesis $H: p \geq \theta$ against an alternative hypothesis $K: p<\theta$ where $\theta$ is a threshold over $(0,1)$ and $p$ is a true probability that $\mathcal{M}$ satisfies $\phi$. Hypothesis testing checks if $H$ is accepted or not based on the randomly sampled paths. For testing a hypothesis $H$, there are two types of errors such as false negative (also known as a type I error) which rejects a true hypothesis $H$ and false positive (also known as a type II error) which accepts a false hypothesis $H$. We can bound an error probability of a false negative error within $\alpha$. Similarly, we can bound an error probability of a false positive error within $\beta$. We call $\alpha$ and $\beta$ as error bounds. The left side of Figure 2 presents the function of probability $L_{p}$ of accepting the hypothesis $H$ as a function of $p$ with the probability of a type I error and type II error as exactly $\alpha$ and $\beta$. However, we want to give similar probability $L_{p}$ of $p=\theta$ and $p=\theta-\epsilon$ for arbitrarily small $\epsilon>0$ for reality. To solve this problem, we introduce an indifference region $\left(p_{1}, p_{0}\right)$ around $\theta$ where $p_{0}=\theta+\delta, p_{1}=\theta-\delta$, and $\delta$ is a half size of indifference region (see right side function in Figure 2). Therefore, instead of testing $H$ against $K$, we use the modified hypothesis

$$
H_{0}: p \geq p_{0}
$$

against the alternative hypothesis

$$
H_{1}: p<p_{1}
$$

If the probability $p$ is in $\left(p_{1}, p_{0}\right)$, then $p$ is sufficiently close to $\theta$ so that we do not care which hypothesis is accepted.

Now, we describe the algorithm of SPRT. First, we obtain a sample path $\sigma_{i}$ of a target system by simulating the target system and model-check if the sample path $\sigma_{i}$ satisfies the given property $\phi$ (see Section II-A). After generating $m$ th 

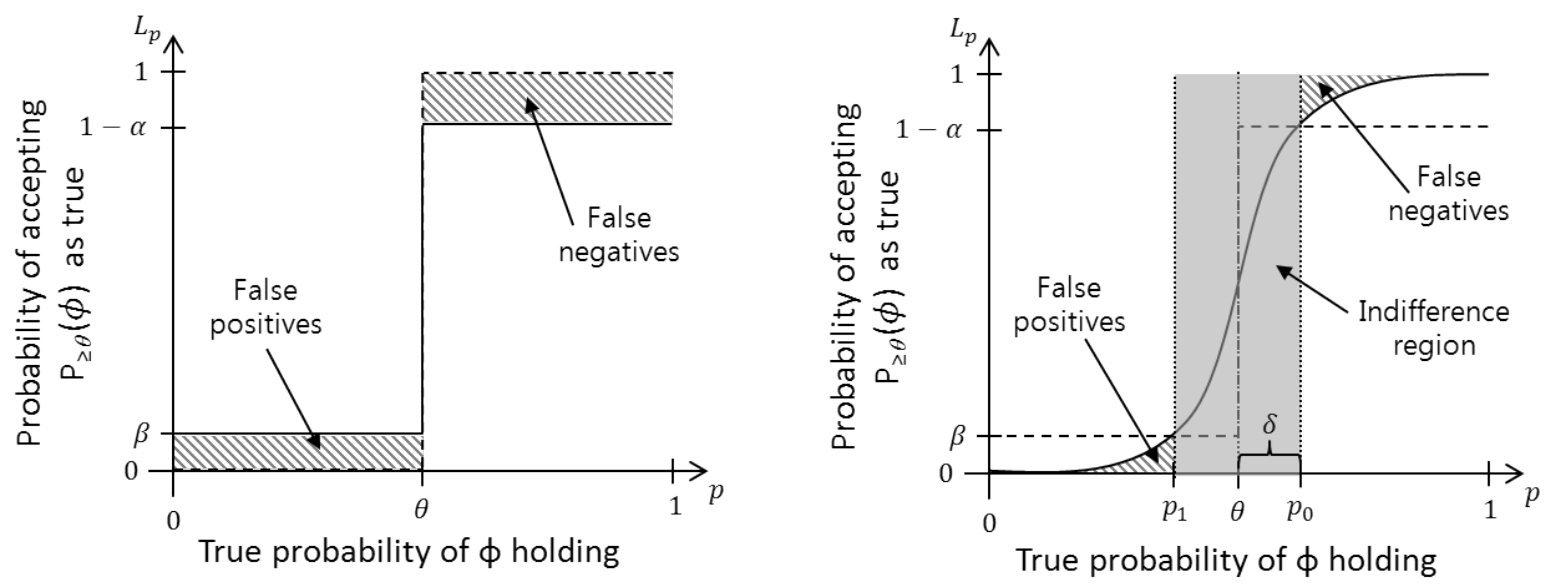

Fig. 2. Function of probability $L_{p}$ of accepting the hypothesis $H: p \geq \theta$ (left side) and function of probability $L_{p}$ of accepting the hypothesis $H_{0}: p \geq p_{0}$ with indifference region (right side).

sample paths of the test, we calculate the quantity

$$
\frac{p_{1 m}}{p_{0 m}}=\prod_{i=1}^{m} \frac{\operatorname{Pr}\left[X_{i}=x_{i} \mid p=p_{1}\right]}{\operatorname{Pr}\left[X_{i}=x_{i} \mid p=p_{0}\right]}=\frac{p_{1}^{d_{m}}\left(1-p_{1}\right)^{m-d_{m}}}{p_{0}^{d_{m}}\left(1-p_{0}\right)^{m-d_{m}}}
$$

where $d_{m}=\sum_{i=1}^{m} x_{i}$ and $x_{i}$ is $i$ th observation of $\sigma_{i} \models \phi . p_{j m}$ is the probability of the sequence $x_{1}, \ldots, x_{m}$ with $\operatorname{Pr}\left[X_{i}=\right.$ $1]=p_{j}$ for $j=0,1$. Therefore, the above quantity makes the ratio of two probabilities, the probability ratio. The hypothesis $H_{0}$ is accepted if

$$
\frac{p_{1 m}}{p_{0 m}} \leq B
$$

and the hypothesis $H_{1}$ is accepted if

$$
\frac{p_{1 m}}{p_{0 m}} \geq A .
$$

Otherwise, we should generate $m+1$ th sample path of the test. $A$ and $B$ are selected to bound error probability $\alpha$ and $\beta$, with $A>B$. In practice, we choose $A=\frac{1-\beta}{\alpha}$ and $B=$ $\frac{\beta}{1-\alpha}$ (detailed description is found in [16], [19]).

Note that SPRT can be imprecise with same indifference region value $\delta$ when the threshold $\theta$ is close to 1 . The reason for the imprecise result of SPRT is due to the limited size of indifference region. For example, if the threshold $\theta$ is 0.99 and $\delta \geq 0.01$, then $p_{0}$ becomes 1 , which causes the denominator of the probability ratio $\frac{p_{1 m}}{p_{0 m}}$ to be 0 when one false sample path occurs, which can cause imprecise result. Therefore, $\delta$ should be very small when $\theta$ is close to 1 , which requires large number of samples.

\section{Bayesian Interval Estimation Testing}

Bayesian interval estimation testing (BIET) is an estimation testing based SMC technique. Estimation testing can approximately compute $p$, the probability that the model $\mathcal{M}$ satisfies the given property $\phi$ expressed by bounded linear temporal logic (BLTL). With $p$, we can determine if the probabilistic bounded linear temporal logic (PBLTL) is satisfied. For that purpose, we use a following statistical estimation testing technique.
BIET [23] dynamically determines the number of sample paths for checking the satisfiability of the model $\mathcal{M}$ with the property $\phi$ during simulation as SPRT does. In Bayes' theorem, we get prior probability using current information first. After obtaining new information, we can obtain posterior probability refining prior probability. BIET uses the Bayes' theorem to determine the number of sample paths of the test.

BIET uses four precision parameter inputs such as a halfsize $\delta^{\prime}$ of an estimation interval which will contain $p$ with high probability, the coverage goal $c$ of the estimation interval, and the parameters $\alpha^{\prime}, \beta^{\prime}$ of the Beta prior. In fact, BIET estimates interval around the probability $p$ instead of estimating $p$, but we regard the mean of the estimated interval as $\hat{p}$, the estimated value of true probability $p$, i.e., the estimated interval is $(\hat{p}-$ $\left.\delta^{\prime}, \hat{p}+\delta^{\prime}\right)$. We call the estimated interval as $\left(t_{0}, t_{1}\right)$. We have a coverage goal such that the probability that the probability satisfying $\mathcal{M} \models \phi$ is in $\left(t_{0}, t_{1}\right)$ should be over the coverage $c \in\left(\frac{1}{2}, 1\right)$. The exact description of the coverage goal is as follows:

$$
\int_{t_{0}}^{t_{1}} f\left(u \mid x_{1}, \ldots, x_{n}\right) d u=c
$$

where $x_{i}$ is $i$ th observation of $\sigma_{i} \models \phi$ for $i=1, \ldots, n$ and $n$ is the number of sample paths. We call the coverage goal as a $100 c$ percent Bayesian interval estimate of $p$. Since BIET uses the Bayes' theorem, we need prior information, i.e., prior density of $p$ to obtain prior probability. For simplicity, we focus on the Beta prior with parameters $\alpha^{\prime}, \beta^{\prime}$.

At $m$ th stage of the test, by Beta prior with $\alpha^{\prime}, \beta^{\prime}$, we can calculate the quantity

$$
\hat{p}=\frac{x+\alpha^{\prime}}{m+\alpha^{\prime}+\beta^{\prime}}
$$

where $x=\sum_{i=1}^{m} x_{i}$ is the number of success sample paths during $m$ number of sample paths. Next, using $t_{0}=\hat{p}-$ $\delta^{\prime}, t_{1}=\hat{p}+\delta^{\prime}$, we can calculate the quantity

$$
\gamma=\int_{t_{0}}^{t_{1}} f\left(u \mid x_{1}, \ldots, x_{m}\right) d u
$$


where $\gamma$ is the coverage of $m$ number of sample paths for checking $\mathcal{M}=\phi$. If $\gamma \geq c$, then BIET stops the simulation and outputs $t_{0}, t_{1}$, and $\hat{p}$. Otherwise, BIET generates $m+1$ th sample path and repeats.

Note that BIET is fast when the estimated probability $\hat{p}$ is close to 0 or 1 [23], whereas BIET is extremely slow (i.e., extremely larger number of samples is required) when $\hat{p}$ is close to $\frac{1}{2}$. With this advantage of BIET, BIET can easily apply the problem for safety critical system since the probability standard of satisfiability for safety critical system should be usually close to 1 or 0 .

\section{HYBRID SMC AlgORITHM}

We develop a hybrid SMC technique to improve efficiency and effectiveness by combining SPRT whose verification speed is fast (i.e., small number of samples is required) and BIET whose verification precision is high (i.e., the number of false positive and false negative results is small) [9]. Algorithm 1 describes how the hybrid SMC technique checks if a target system model $\mathcal{M}$ satisfies a property $\phi$ in BLTL for a probability threshold $\theta^{1}$ with precision parameters $\operatorname{par}_{S}$ for SPRT and $\operatorname{par}_{B}$ for BIET. The algorithm first applies SPRT multiple times with dynamically increasing probability threshold $\theta_{S P R T}$ until a verification result is 'reject' (lines 1518) or $\theta_{S P R T}$ becomes larger than or equal to a threshold $t h_{S 2 B}$ where $0.5<t h_{S 2 B} \leq \theta$ (lines 5-20). If $\theta_{S P R T}$ becomes larger than or equal to $t h_{S 2 B}$, the algorithm applies BIET to obtain a precise verification result (lines 21-34).

The detail of the algorithm is as follows. First, the algorithm calls $S P R T() m_{S}$ times (lines 6-10), which applies SPRT to $\mathcal{M}$ with regard to $\phi$ and $\theta_{S P R T}$ with $\operatorname{par}_{S}$ (line 8). A result of $S P R T()$ is 'accept' (i.e., 1) or 'reject' (i.e., 0). After $m_{S}$ trials of $S P R T()$, the algorithm calculates an average accept decision value accept $t_{\text {avg }}$ over the $m_{S}$ trials (line 11). If accept $_{\text {avg }}$ is less than a user-given accept decision threshold $t h_{\text {acpt }}$, the algorithm decides that the verification result of $\mathcal{M} \models P_{\geq \theta}(\phi)$ is 'reject' (line 16) and terminates (line 18). Otherwise (i.e., accept $t_{a v g} \geq t h_{a c p t}$ ), the algorithm increases $\theta_{S P R T}$ from the initial value 0.5 (line 3 ) to $0.75,0.875,0.9375$ and so on (line 14) until $\theta_{S P R T}$ becomes larger than or equal to $t h_{S 2 B}$ through the while loop in lines 5-20.

If $\theta_{S P R T}$ becomes larger than or equal to a user-given probability threshold $t h_{S 2 B}$ for applying BIET, the algorithm calls $B I E T()$ for $m_{B}$ times (lines 23-27), which applies BIET to $\mathcal{M}$ for $\phi$ with precision parameters $\operatorname{par}_{B}$ (line 25). Based on the estimated probability $p$ obtained from $B I E T()$, the algorithm calculates an average estimated probability $p_{a v g}$ over the $m_{B}$ trials (line 28). If $p_{a v g}$ is greater than or equal to $\theta$, then the algorithm decides that the verification result is 'accept' (lines 29-30); 'reject', otherwise (lines 31-32).

Note that the hybrid SMC algorithm can save a large amount of time cost compared to BIET, if a probability for $\mathcal{M}$ to satisfy $\phi$ is far from a given probability threshold $\theta$. For example, if the probability is less than 0.5 , the algorithm terminates after

\footnotetext{
${ }^{1}$ We assume that $\theta$ is close to 1 , since we develop a hybrid SMC algorithm for safety critical systems whose reliability criteria are very high and, thus, requirement properties are given with high threshold values.
}

executing $S P R T()$ only once without executing $B I E T()$ whose time cost is very high (see Table III). The algorithm executes $B I E T()$ if the probability is close to $\theta$ (which is usually close to 1 for requirement properties of safety critical systems), which is necessary since SPRT becomes imprecise when $\theta$ is close to 1 (Section II-C).

\section{TARget SAFETy CRITICAL SyStems}

This section presents an overview of the following three safety critical systems in automobile domain:

- Automatic transmission control system (ATCS) [13]

- Anti-lock braking system (ABS) [1]

- Fault-tolerant fuel control system (FFCS) [12]

We selected these systems as target systems to apply SPRT, BIET, and the hybrid statistical model checking (SMC) technique (Section III) for the following reasons:

- These three automobile systems [12], [1], [13] are safety critical systems whose reliability is very important. Many researchers are working to address the reliability issues on safety critical systems [2], [14], [18].

- The three automobile systems are complex real-world applications, not a toy example such as ones in probabilistic symbolic model checker (PRISM) [11] benchmarks.

- Simulink/stateflow models of the three automobile systems are publicly available in Matlab R2010a. Thus, it is convenient to build a prototype tool for the SMC techniques by using a Simulink/stateflow simulator.

\section{A. Automatic Transmission Control System}

An automatic transmission control system (ATCS) changes an engine gear automatically to drive smoothly. A main task of ATCS is to select a proper engine gear. As described in Figure 3, ATCS receives inputs regarding car speed, throttle, brake pressure (and engine RPM as a feedback) and calculates an engine RPM and a gear state. ATCS consists of a torque converter and a transmission control unit. The torque converter calculates an impeller torque value to deliver power to control the engine RPM based on the engine RPM and the gear state (i.e., if the impeller torque increases/decreases, the engine RPM increases/decreases). With the sensor inputs on car speed, throttle, and brake pressure, transmission control unit (TCU) selects a proper gear. Based on throttle and brake pressure values, TCU calculates a up-threshold and a downthreshold of a car speed. If a current car speed is greater than the up-threshold or less than the down-threshold, TCU changes the engine gear to keep the engine RPM in safe range.

The size and complexity of the Simulink/stateflow ATCS model in terms of the Halstead metrics [5] are described in Table I. We counted each atomic block (i.e., a module of a mathematical function or control logic) as an operator and each input of an atomic block as an operand of the Simulink/stateflow ATCS model. The automatically generated $\mathrm{C}$ code from the model has 2353 LOC in 71 functions.

A requirement property for ATCS is that the engine RPM is less than 6000 for 30 seconds ${ }^{2}$ should be greater than or

\footnotetext{
${ }^{2}$ We set the time duration to monitor as 30 seconds, since a default simulation time of the Simulink model of ATCS included in Matlab R2010a is 30 seconds.
} 


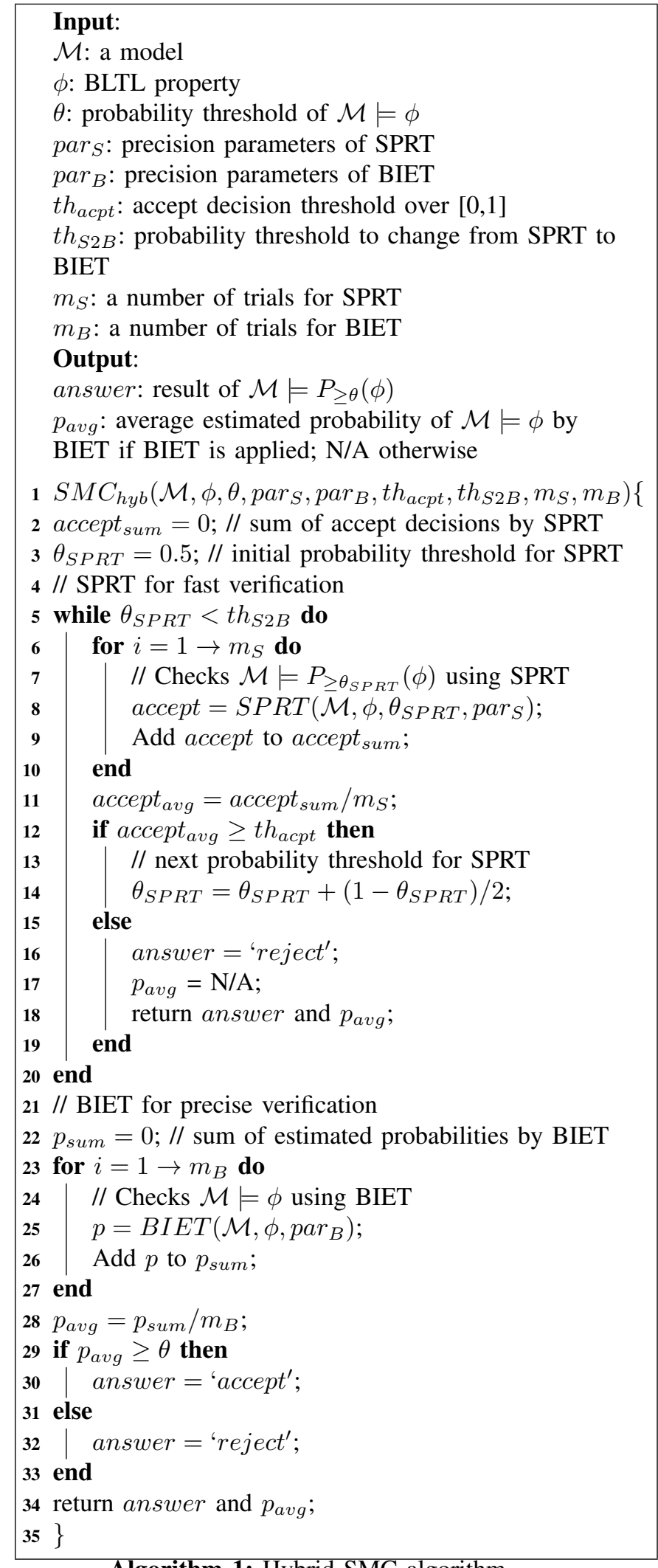

Algorithm 1: Hybrid SMC algorithm

equal to probability $\theta$. The property is important in real world, because if the engine RPM is constantly over 6000 , the engine becomes overheated and can be damaged. The property can

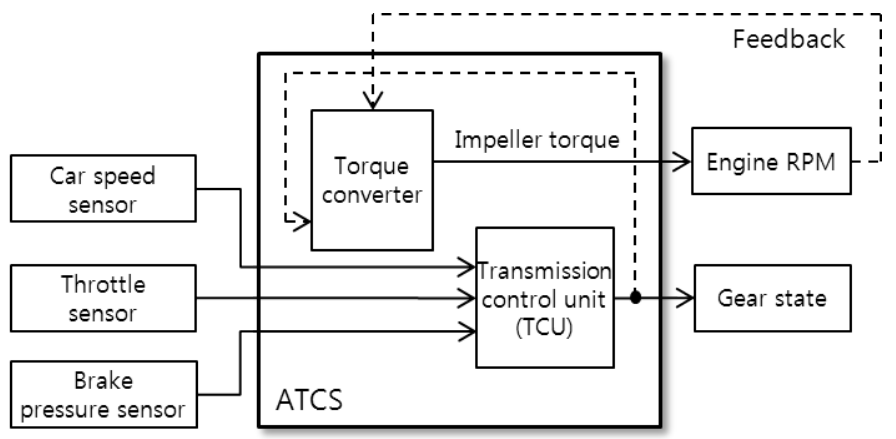

Fig. 3. Block diagram of ATCS

be expressed in PBLTL as follows:

$$
P_{\geq \theta}\left[G^{30}(\text { engine } R P M<6000)\right]
$$

\section{B. Anti-lock Braking System}

An anti-lock braking system (ABS) is a safety system that repeatedly increases and decreases the brake pressure to allow the wheels of a car to interact with the road surface continuously as directed by a driver while braking. Thus, ABS can prevent the wheels from locking up and avoid skidding, which can enhance the safety of driving by improving vehicle control and decreasing stopping distances. As described in Figure 4, ABS has the following three sensors: a car speed sensor, a wheel speed sensor, and a brake pedal sensor. ABS receives data from these sensors and generates the brake pressure and slip as outputs, where slip indicates how properly a wheel of a car is controlled. ABS consists of a bangbang controller and a hydraulic control unit. The bang-bang controller receives data from the three input sensors and commands the hydraulic control unit to increase/decrease the brake pressure. In addition, when the brake pedal is pressed, the bang-bang controller calculates slip as follows:

$$
\text { slip }=1-\frac{\text { wheelspeed }}{\text { carspeed }}
$$

When the wheel speed is equal to the car speed, slip becomes zero. When the wheel speed is zero (i.e., the wheel is locked), slip becomes one, which means that the driver loses control of the car. There is an ideal slip value (which is 0.2) that maximizes the adhesion between the wheel and the road and minimizes the stopping distance with available friction. The bang-bang controller tries to adjust slip close to the ideal slip value by controlling the hydraulic control unit.

The size and complexity of the Simulink/stateflow ABS model in terms of the Halstead metrics are described in Table I. The automatically generated C code from the model has 3443 LOC in 27 functions.

A requirement property for ABS is that for 17 seconds ${ }^{3}$, when the brake pedal is pressed and the car speed is greater than $5 \mathrm{~m} / \mathrm{s}$, slip is less than or equal to 0.9 , should be larger

\footnotetext{
${ }^{3}$ We set the time duration to monitor as 17 seconds, since a default simulation time of the Simulink model of ABS included in Matlab R2010a is 17 seconds.
} 


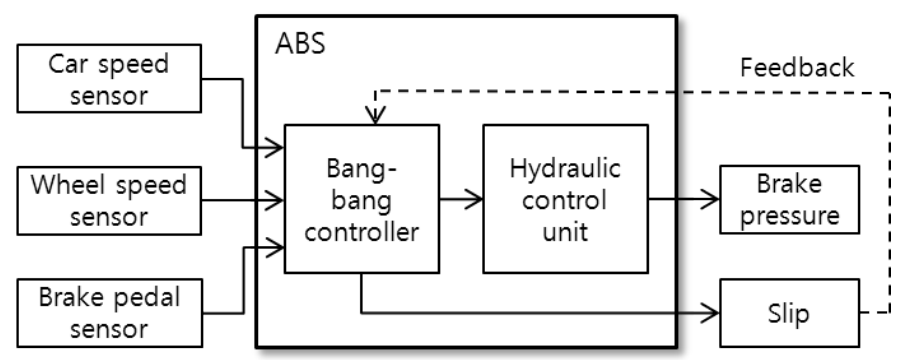

Fig. 4. Block diagram of ABS

than or equal to probability $\theta$. The property is important in real world, because if slip becomes close to 1 when a car is driving, the wheel can be locked and a driver loses control of the car. The property can be expressed in PBLTL as follows:

$$
P_{\geq \theta}\left[G^{17}((\text { brakepressed } \wedge \text { carspeed }>5) \rightarrow \text { slip } \leq 0.9)\right]
$$

\section{Fault-tolerant Fuel Control System}

Figure 5 is an overall diagram of a fault-tolerant fuel control system (FFCS). FFCS [12] controls a fuel rate to inject fuel based on sensor data for best performance, detects a sensor fault, and shuts down an engine for safety in the presence of multiple sensor failures. FFCS has the following four sensors: throttle angle sensor, speed sensor, exhaust gas oxygen (EGO) sensor, and manifold absolute pressure (MAP) sensor. FFCS receives these four sensor inputs and generates a proper fuel rate and an air-fuel ratio. FFCS consists of the following three components: a fuel rate controller, an airfuel ratio calculator, and a sensor failure detector. The fuel rate controller receives the four sensor data and calculates a proper fuel rate to make the air-fuel ratio optimal (i.e., 14.6). The air-fuel ratio calculator receives EGO sensor data and a fuel rate and calculates the air-fuel ratio. The sensor failure detector receives all four sensor data and controls the fuel rate controller to increase/decrease the fuel rate in the presence of a single sensor fault or shuts down the engine if multiple sensors fail, since the air-fuel ratio cannot be controlled with failures of multiple sensors.

The size and complexity of the Simulink/stateflow FFCS model in terms of the Halstead metrics are described in Table I. The automatically generated $\mathrm{C}$ code from the model has 8266 LOC in 222 functions.

A requirement property for FFCS is that the fuel rate does not become zero for one second in 100 seconds should be greater than equal to probability $\theta$. The property is crucial in a real world, because if the fuel rate is zero for one second, then the engine stops and can cause a serious accident. This property can be expressed by PBLTL as follows [23]:

$$
P_{\geq \theta}\left[\neg\left(F^{100} G^{1}(\text { fuelrate }=0)\right)\right]
$$

\section{EXPERIMENTAL STUDY}

We have applied SPRT, BIET, and the hybrid SMC technique to ATCS, ABS, and FFCS with precision parameters as independent variables to check if these target systems satisfy

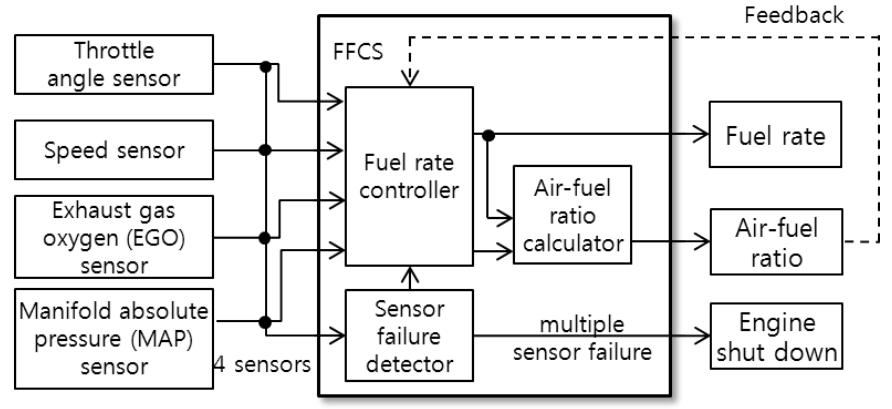

Fig. 5. Block diagram of FFCS

the given requirement properties in PBLTL. In addition, we have compared the results of the hybrid SMC technique with the results of SPRT and BIET. We used Simulink/stateflow models of the three systems included in the Matlab R2010a example directory.

\section{A. Experiment Setup}

1) Environment Setup: We used the input value generation modules provided in the Simulink/stateflow models of FFCS, ATCS, and ABS without modification. In addition, we built the stochastic environments for the three automobile systems as follows:

- ATCS: we built a stochastic environment to ATCS by modeling a random delay to transfer the engine RPM value from the engine to the torque converter. ${ }^{4}$ This random delay is modeled by exponential distribution [10]. We selected a 'passing maneuver' scenario from the options of the ATCS model, which simulates a situation that a driver opens the throttle $100 \%$ after 15 seconds. We utilize the following four delay rates (i.e., mean delay times of transmission in seconds) $\lambda \in\{0.01,0.02,0.03,0.04\}$.

- ABS: we built a stochastic environment of ABS that generates random delay to the command from the bangbang controller to the hydraulic control unit. ${ }^{5}$ The random delay of the command is modeled by exponential distribution [10]. We use a model of ABS representing a single wheel, which can be duplicated multiple times to create a model for a multi-wheel vehicle. We utilize the following four delay rates (in seconds) $\lambda \in$ $\{0.001,0.003,0.005,0.007\}$.

- FFCS: we built a stochastic environment model for FFCS that generates random faults at the EGO, MAP, and speed sensors as Zuliani et al. [23] did. The random faults are modeled by three independent Poisson processes with different arrival rates [17]. We assume one fault event remains for one second. When a fault event occurs in a sensor, FFCS remains in a failure mode in one second and returns to a normal mode. We utilize the following four inter-arrival fault rates (i.e., mean inter-arrival times

\footnotetext{
${ }^{4}$ This random delay is a real factor, not an artificial one. ATCS has an electronic circuit to deliver data from one sub-component to another and the data transfer can be delayed non-deterministically due to non-deterministic scheduling and bus contention among multiple sub-component.

${ }^{5}$ This random delay is a real factor for the similar reason of the one in ATCS.
} 
TABLE I

Size AND COMPLEXITy OF THE SimULink MOdels of ATCS, ABS, AND FFCS IN HALSTEAD METRICS

\begin{tabular}{c||c|c|c|c||c|c|c|c|c}
\hline \hline $\begin{array}{c}\text { Target } \\
\text { system }\end{array}$ & $\begin{array}{c}N_{1}: \text { \# of } \\
\text { operators }\end{array}$ & $\begin{array}{c}N_{2}: \# \text { of } \\
\text { operands }\end{array}$ & $\begin{array}{c}n_{1}: \# \text { of } \\
\text { distinct } \\
\text { operators }\end{array}$ & $\begin{array}{c}n_{2}: \# \text { of } \\
\text { distinct } \\
\text { operands }\end{array}$ & $\begin{array}{c}N \text { :program } \\
\text { length } \\
\left(=N_{1}+N_{2}\right)\end{array}$ & $\begin{array}{c}n \text { : program } \\
\text { vocabulary } \\
\left(=n_{1}+n_{2}\right)\end{array}$ & $\begin{array}{c}V \text { : program } \\
\text { volume } \\
(N \times \operatorname{logn})\end{array}$ & $\begin{array}{c}D: \text { program } \\
\text { difficulty } \\
\left(=n_{1} / 2 \times N_{2} / n_{2}\right)\end{array}$ & $\begin{array}{c}E: \text { program } \\
\text { effort } \\
(=\mathrm{D} \times \mathrm{V})\end{array}$ \\
\hline \hline ATCS & 31 & 46 & 27 & 39 & 77 & 66 & 465.4 & 15.9 & 7410.9 \\
\hline ABS & 27 & 36 & 19 & 36 & 63 & 55 & 364.2 & 9.5 & 3460.1 \\
\hline FFCS & 65 & 111 & 35 & 94 & 176 & 129 & 1234.0 & 20.7 & 25500.0 \\
\hline \hline
\end{tabular}

of sensor fault) to the three sensors: $(3,7,8),(10,8,9)$, $(20,10,20)$ and $(30,30,30)$.

2) Precision Parameter Setup: We use the following precision parameters for SPRT and BIET:

- SPRT:

- a half-size of indifference region $\delta \in$ $\{0.01,0.03,0.05\}$

- error bounds $\alpha, \beta \in\{0.1,0.01,0.001\}$

- BIET $^{6}$ :

- interval coverage $c \in\{0.9,0.99,0.999\}$

- a half-size of estimation interval $\delta^{\prime} \in$ $\{0.01,0.03,0.05\}$

- parameters of Beta prior $\alpha^{\prime}=\beta^{\prime}=1$ (since we assume the prior density to be a uniform density over $(0,1))$

We performed each experiment five times to obtain average verification result over $[0,1]$ regarding if the hypothesis $H_{0}$ is accepted where $H_{0}$ : a probability for $\mathcal{M}$ to satisfy $\phi$ is greater than or equal to $\theta+\delta$. For the experiments, we used $\theta \in\{0.5,0.7,0.9,0.99\}$. In addition, we measured the total verification time and total number of samples for each experiment.

For the hybrid SMC technique, we set $\theta=0.99$. This is because the hybrid SMC technique targets safety critical systems which require high reliability, which can be specified with PBLTL with high $\theta$ values. We use the following precision parameters which are similar to those of the SPRT and BIET experiments:

- precision parameters for SPRT $\operatorname{par}_{S}: \delta \in \in$ $\{0.01,0.03,0.05\}, \alpha, \beta \in\{0.1,0.01,0.001\}$.

- precision parameters for BIET $\operatorname{par}_{B}: \quad c \in$ $\{0.9,0.99,0.999\}, \delta^{\prime} \in\{0.01,0.03,0.05\}, \alpha^{\prime}=\beta^{\prime}=1$.

- threshold for accept decision over $[0,1] t h_{a c c}=0.5$

- the probability threshold to apply BIET instead of SPRT $t h_{S 2 B}=0.95$

- the number of trials for SPRT $m_{S}=5$

- the number of trials for BIET $m_{B}=5$

3) Experiment Platform: We built a statistical model checker as a Matlab module, which executes the Simulink/stateflow models for FFCS, ATCS, and ABS and monitors inputs and outputs of the models to check if $\phi$ is satisfied on a current sample path. After each execution of

\footnotetext{
${ }^{6}$ Our parameters are similar to those of Zuliani et al. [23], where they use interval coverage $c$ as 0.99 and 0.999 and half-size of estimation interval $\delta$ as 0.01 and 0.05 . To identify tendency of the experimental results more, we used more parameters.
}

the models, the SMC module calculates a required number of samples dynamically based on the precision parameters and the number of success/fail samples generated so far. If a number of the generated samples reaches the required number, the SMC module generates a verification result. The SMC module for SPRT is around 80 lines long. The SMC module for BIET is around 70 lines long. The hybrid SMC module is around 200 lines long. We used Matlab R2010a for the experiments. All experiments were performed on 64 bit Windows 7 Professional equipped with a $3 \mathrm{GHz}$ Intel processor and 16 gigabytes of memory.

\section{B. Results of SPRT and BIET}

Tables II and III describe the experiment results of applying SPRT with $\delta=0.03$ and BIET to ATCS respectively when the delay rate $\lambda=0.03 .{ }^{7}$ In Tables II and III, $n$ is a total number of required sample execution paths for the five trials and time is total verification time taken for the five trials in seconds. acpt in Table II is an average result over $[0,1]$ regarding the hypothesis $H_{0}$ where 0 is 'reject' and 1 is 'accept'. $\hat{p}$ in Table III is an estimated probability for $\mathcal{M} \models \phi$.

Table II shows that the probability for ATCS with $\lambda=0.03$ and $\delta=0.03$ to satisfy the requirement property $\phi$ $\left(=G^{30}(\right.$ engine $\left.R P M<6000)\right)$ is between 0.7 and 0.9 . This is because acpts are 1.0 when $\theta \leq 0.7$ while acpts are 0.0 when $\theta=0.9$ in Table II (the verification result of SPRT with a high $\theta$ value like 0.99 should not be trusted due to the characteristics of SPRT [21]).

In addition, we can conclude that the probability is close to 0.9 , since $n$ of SPRT increases as $\theta$ increases from 0.5 to 0.9 and decreases sharply from 0.9 to 0.99 . For example, Table II shows that $n$ becomes $110,215,343$, and 18 as $\theta$ becomes 0.5 , $0.7,0.9$, and 0.99 with $\alpha=\beta=0.1$. This tendency of $n$ indicates that the true probability for ATCS with $\lambda=0.03$ to satisfy $\phi$ is close to 0.9 , since SPRT requires a large number of sample paths to check a given hypothesis $H_{0}$ if a true probability is close to $\theta$ [21]. Furthermore, the verification result of BIET coincides with that of SPRT, since Table III shows that the estimated probability $\hat{p}$ is between 0.8544 (with $c=0.9$ and $\delta^{\prime}=0.03$ ) and 0.9000 (with $c=0.99$ and $\delta^{\prime}=0.03$ ).

For the verification speed, Tables II and III show that SPRT is much faster than BIET. For example, the maximum time spent by SPRT in Table II is 636.7 seconds with $\theta=0.9$ and

\footnotetext{
${ }^{7}$ Due to page limit, we cannot describe full experiment data in the paper. Full experiment data of applying SPRT and BIET to ATCS, ABS, and FFCS is available at http://pswlab.kaist.ac.kr/data/issre2012-expr-results.zip
} 
TABLE II

EXPERIMENT RESULT OF SPRT FOR ATCS WITH $\lambda=0.03$ AND $\delta=0.03$ FOR THE FIVE TRIALS

\begin{tabular}{|c|c|c|c|c|c|c|c|c|c|c|c|c|}
\hline \multirow{3}{*}{$\alpha, \beta$} & \multicolumn{12}{|c|}{ threshold $\theta$} \\
\hline & \multicolumn{3}{|c|}{0.5} & \multicolumn{3}{|c|}{0.7} & \multicolumn{3}{|c|}{0.9} & \multicolumn{3}{|c|}{0.99} \\
\hline & $n$ & acpt & time & $n$ & acpt & time & $n$ & acpt & time & $n$ & $a c p t$ & time \\
\hline 0.1 & 110 & 1.0 & 69.9 & 215 & 1.0 & 143.9 & 343 & 0.0 & 221.8 & 18 & 1.0 & 12.6 \\
\hline 0.01 & 270 & 1.0 & 171.0 & 375 & 1.0 & 301.1 & 410 & 0.0 & 347.1 & 41 & 1.0 & 27.1 \\
\hline 0.001 & 395 & 1.0 & 249.0 & 563 & 1.0 & 361.1 & 985 & 0.0 & 636.7 & 45 & 1.0 & 30.2 \\
\hline
\end{tabular}

TABLE III

EXPERIMENT RESULT OF BIET FOR ATCS WITH $\lambda=0.03$ FOR THE FIVE TRIALS

\begin{tabular}{c||r|r|r|r|r|r||r|r|r}
\hline \hline \multirow{2}{*}{\multicolumn{1}{c||}{$\delta^{\prime}$}} & \multicolumn{9}{c}{ interval coverage $c$} \\
\cline { 2 - 10 } & \multicolumn{4}{c||}{0.9} & \multicolumn{1}{c}{0.99} & \multicolumn{3}{c}{0.999} \\
\cline { 2 - 10 } & $n$ & $\hat{p}$ & time & $n$ & $\hat{p}$ & time & $n$ & $\hat{p}$ & time \\
\hline \hline 0.05 & 630 & 0.8594 & 416.6 & 1550 & 0.8654 & 1011.9 & 2665 & 0.8636 & 1753.2 \\
\hline 0.03 & 1845 & 0.8544 & 1208.6 & 3340 & 0.9000 & 2181.1 & 6475 & 0.8805 & 4356.5 \\
\hline 0.01 & 14150 & 0.8810 & 9551.8 & 36540 & 0.8740 & 26281.2 & 58870 & 0.8762 & 42945.1 \\
\hline \hline
\end{tabular}

$\alpha=\beta=0.001$, which is less than time costs of BIET in Table III except when BIET is applied with low precision parameters $\delta^{\prime}=0.05$ and $c=0.9$ (416.6 seconds).

Thus, if a given PBLTL formula has a high $\theta$ value like 0.99 , it is a good idea to apply SPRT first with low $\theta$ values (SPRT result with high $\theta$ value should not be trusted) in hope of eliminating the need to apply BIET. For example, suppose that we should check $P_{\geq \theta}\left[G^{30}\right.$ (engine $\left.\left.R P M<6000\right)\right]$ for ATCS with $\lambda=0.03$ and $\theta=0.99$. With $\alpha=\beta=0.1$, SPRT takes 435.6 seconds in total $(=69.9+143.9+221.8)$ to conclude that ATCS does not satisfy the given PBLTL formula with $\theta=0.99$ by checking cases with $\theta$ as $0.5,0.7$, and 0.9 in order (Table II); the verification result with $\theta=0.9$ is 'reject', which consequently makes the result with $\theta=0.99$ as 'reject'. However, if we apply BIET, we will obtain the same verification result with higher time cost except a case with $\delta^{\prime}=0.05$ and $c=0.9$ (416.6 seconds (Table III)). The hybrid SMC technique (Algorithm 1) is developed to utilize this observation for precise and fast verification.

\section{Results of the Hybrid SMC Technique}

Tables IV-VI present the experiment results of the hybrid SMC technique on ATCS, ABS, and FFCS for $\theta=0.99$ with $\delta=0.03, \delta^{\prime}=0.01$, and $c=0.99$, respectively. $n$ is a total number of sample paths required by SPRT and BIET in the hybrid algorithm for each experiment. $\hat{p}$ is an estimated probability obtained by BIET for each experiment. If BIET is not applied because SPRT rejects a hypothesis $H_{0}$ before reaching $t h_{S 2 B}$, then $\hat{p}$ is N/A. acpt is a result over $[0,1]$ regarding the hypothesis $H_{0}$ where 0 is 'reject' and 1 is 'accept'. time is total verification time taken for each expriperettcation secondsults: For ATCS, Table IV shows that the corresponding hypothesis $H_{0}$ with $\theta=0.99$ is accepted for two delay rates $\lambda \in\{0.01,0.02\}$ (i.e., $\mathcal{M}=$ $P_{\geq \theta}\left[G^{30}(\right.$ engine $\left.R P M<6000)\right]$ and rejected for delay rates $\lambda \in\{0.03,0.04\}$. For the experiments with $\lambda \in\{0.03,0.04\}$, SPRT rejected $H_{0}$ and $B I E T$ was not applied; thus, corresponding $\hat{p} s$ are marked as 'N/A'. This result coincides with the results of SPRT and BIET, since SPRT concludes that ATCS with $\lambda=0.03$ does not satisfy the PBLTL formula with $\theta=0.9$ (i.e., acpts are all 0.0 in Table II) and BIET concludes that the probability for ATCS with $\lambda=0.03$ to satisfy $G^{30}($ engine $R P M<6000)$ is between 0.8544 and 0.9000 (Section V-B).

An interpretation of this result is that ATCS may not operate correctly if an engine RPM value is transferred from the engine to the torque converter with long delay (i.e., delay rate $\lambda$ in exponential distribution is larger than or equal to 0.03 seconds), since long delay of the data transfer can prevent ATCS from operating promptly. In addition, we can obtain a practical implication that, to achieve required high reliability specified by the PBLTL formula with $\theta=0.99$, ATCS should use a data-transfer component that transfers data from the engine to the torque converter with delay rate $\lambda \leq 0.02$ or revise the ATCS design to satisfy the PBLTL formula with $\theta=0.99$ even with long delay of the data transfer.

Similarly, for ABS, Table V shows that the corresponding hypothesis $H_{0}$ with $\theta=0.99$ is accepted for delay rate $\lambda=0.001$ (i.e., $\mathcal{M} \models P_{\geq \theta}\left[G^{17}\right.$ ((brakepressed $\wedge$ carspeed $>$ $5) \rightarrow$ slip $\leq 0.9)]$ ), and is rejected for larger delay rates. For FFCS, Table VI shows that the corresponding hypothesis $H_{0}$ with $\theta=0.99$ is accepted for fault ratios $(20,10,20)$ (except $\alpha=\beta=0.001$ ) and $(30,30,30)$ (i.e., $\mathcal{M} \models$ $P_{\geq \theta}\left[\neg\left(F^{100} G^{1}(\right.\right.$ fuelrate $\left.\left.\left.=0)\right)\right]\right)$, and is rejected for more frequent fault ratios (3.7,8) and $(10,89 \mathrm{~S}$ MC technique shows an order of magnitude faster verification speed compared to BIET for the experiments where the probability for $\mathcal{M} \models \phi$ is less than $t h_{S 2 B} .{ }^{8}$ For example, for ATCS with $\lambda=0.03$, the hybrid technique spent 698.9 seconds (with $\alpha=\beta=0.1, \delta=0.03$, $\delta^{\prime}=0.01$, and $c=0.99$ ) to 6020.4 seconds (with $\alpha=\beta=0.001$, $\delta=0.03, \delta^{\prime}=0.01$, and $c=0.99$ ) (Table IV), while BIET spent 26281.2 seconds for the same precision parameters (i.e., $\delta^{\prime}=$

\footnotetext{
${ }^{8}$ Comparison between the verification speed of the hybrid technique and that of SPRT is not meaningful, since SPRT result is imprecise for a large $\theta$ value like 0.99 .
} 
TABLE IV

EXPERIMENT RESULT OF THE HYBRID SMC FOR ATCS WITH $\theta=0.99, \delta=0.03, \delta^{\prime}=0.01, c=0.99$

\begin{tabular}{|c|c|c|c|c|c|c|c|c|c|c|c|c|c|c|c|c|}
\hline \multirow{3}{*}{$\alpha, \beta$} & \multicolumn{16}{|c|}{ delay rate $\lambda$ from engine to torque convertor } \\
\hline & \multicolumn{4}{|c|}{0.01} & \multicolumn{4}{|c|}{0.02} & \multicolumn{4}{|c|}{0.03} & \multicolumn{4}{|c|}{0.04} \\
\hline & $n$ & $\hat{p}$ & acpt & time & $n$ & $\hat{p}$ & acpt & time & $n$ & $\hat{p}$ & acpt & time & $n$ & $\hat{p}$ & $a c p t$ & time \\
\hline 0.1 & 1710 & 0.9956 & 1 & 1256.1 & 1710 & 0.9956 & 1 & 1173.7 & 1066 & $N / A$ & 0 & 698.9 & 1334 & $N / A$ & 0 & 858.9 \\
\hline 0.01 & 2315 & 0.9956 & 1 & 1740.8 & 2315 & 0.9956 & 1 & 1642.6 & 4795 & $N / A$ & 0 & 3081.9 & 2946 & $N / A$ & 0 & 1884.6 \\
\hline 0.001 & 2905 & 0.9956 & 1 & 2320.2 & 2905 & 0.9956 & 1 & 2102.7 & 7804 & $N / A$ & 0 & 6020.4 & 3833 & $N / A$ & 0 & 2952.4 \\
\hline
\end{tabular}

TABLE V

EXPERIMENT RESULT OF HYBRID SMC FOR ABS WITH $\theta=0.99, \delta=0.03, \delta^{\prime}=0.01, c=0.99$

\begin{tabular}{|c|c|c|c|c|c|c|c|c|c|c|c|c|c|c|c|c|}
\hline \multirow{3}{*}{$\alpha, \beta$} & \multicolumn{16}{|c|}{ delay rate $\lambda$ from bang-bang controller to hydraulic control unit } \\
\hline & \multicolumn{4}{|c|}{0.001} & \multicolumn{4}{|c|}{0.003} & \multicolumn{4}{|c|}{0.005} & \multicolumn{4}{|c|}{0.07} \\
\hline & $n$ & $\hat{p}$ & acpt & time & $n$ & $\hat{p}$ & acpt & time & $n$ & $\hat{p}$ & acpt & time & $n$ & $\hat{p}$ & acpt & time \\
\hline 0.1 & 1814 & 0.9953 & 1 & 986.5 & 6511 & 0.9826 & 0 & 2905.9 & 8247 & 0.9773 & 0 & 3854.4 & 952 & $N / A$ & 0 & 382.4 \\
\hline 0.01 & 2417 & 0.9953 & 1 & 1344.8 & 8006 & 0.9806 & 0 & 3619.4 & 9151 & 0.9770 & 0 & 4290.1 & 2238 & $N / A$ & 0 & 890.2 \\
\hline 0.001 & 3179 & 0.9950 & 1 & 1815.3 & 8541 & 0.9810 & 0 & 3906.1 & 9326 & 0.9791 & 0 & 4334.0 & 3684 & $N / A$ & 0 & 1465.5 \\
\hline
\end{tabular}

TABLE VI

EXPERIMENT RESULT OF HYBRID SMC FOR FFCS WITH $\theta=0.99, \delta=0.03, \delta^{\prime}=0.01, c=0.99$

\begin{tabular}{|c|c|c|c|c|c|c|c|c|c|c|c|c|c|c|c|c|}
\hline \multirow{3}{*}{$\alpha, \beta$} & \multicolumn{16}{|c|}{ sensor fault rates } \\
\hline & \multicolumn{4}{|c|}{$(3,7,8)$} & \multicolumn{4}{|c|}{$(10,8,9)$} & \multicolumn{4}{|c|}{$(20,10,20)$} & \multicolumn{4}{|c|}{$(30,30,30)$} \\
\hline & $n$ & $\hat{p}$ & acpt & time & $n$ & $\hat{p}$ & acpt & time & $n$ & $\hat{p}$ & acpt & time & $n$ & $\hat{p}$ & acpt & time \\
\hline 0.1 & 1299 & $N / A$ & 0 & 3359.6 & 14442 & 0.9575 & 0 & 36399.3 & 3180 & 0.9920 & 1 & 7990.0 & 2121 & 0.9944 & 1 & 5362.0 \\
\hline 0.01 & 5369 & $N / A$ & 0 & 13893.4 & 14130 & 0.9620 & 0 & 35894.1 & 4651 & 0.9906 & 1 & 11786.0 & 3747 & 0.9926 & 1 & 9556.4 \\
\hline 0.001 & 7320 & $N / A$ & 0 & 19059.9 & 16010 & 0.9592 & 0 & 41014.6 & 5809 & 0.9895 & 0 & 14792.1 & 3512 & 0.9939 & 1 & 9017.2 \\
\hline
\end{tabular}

$0.01, c=0.99)$ (Table III). The hybrid technique is much faster than BIET for ATCS with $\lambda=0.03$, since SPRT of the hybrid technique concludes that ATCS with $\lambda=0.03$ does not satisfy the PBLTL formula with $\theta_{S P R T}=0.9375$. Since $\theta_{S P R T}=$ $0.9375<t h_{S 2 B}=0.95$, the hybrid technique does not apply BIET and conclude that ATCS with $\lambda=0.03$ does not satisfy the given PBLTL formula with $\theta=0.99$. As BIET takes an order of magnitude larger time cost than SPRT (Tables II-III), the hybrid technique can reduce a large amount of time cost by removing the time cost of BIET.

However, for the experiments where the probability for $\mathcal{M} \models \phi$ is larger than $t h_{S 2 B}$, the hybrid technique shows slower verification speed compared to BIET. For example, for ATCS with $\lambda=0.02$, the hybrid technique spent $1173.7 \mathrm{sec}$ onds (with $\alpha=\beta=0.1, \delta=0.03, \delta^{\prime}=0.01$, and $c=0.99$ ) to 2102.7 seconds (with $\alpha=\beta=0.001, \delta=0.03, \delta^{\prime}=0.01$, and $c=0.99$ ) (Table IV), while BIET spent 820.1 seconds for the same precision parameters (i.e., $\delta^{\prime}=0.01$ and $c=0.99$ ) (see http:// pswlab.kaist.ac.kr/data/issre2012-expr-results.zip). This larger time cost of the hybrid technique is due to the additional applications of SPRT for $\theta_{S P R T} \in\{0.5,0.75,0.875,0.9375\}$.

For ABS and FFCS, we make similar observations to the experiments for ATCS. For the cases where the probability for $\mathcal{M}=\phi$ is less than $t h_{S 2 B}$, the hybrid technique is much faster than BIET. For the other cases, the hybrid technique is slower than BIET.

\section{A. Effective and Efficient Hybrid SMC Technique}

Through the empirical evaluation of the hybrid statistical model checking technique on ATCS, ABS, and FFCS, we found that the hybrid technique is faster and more accurate than a single SMC technique (Section V-C). This improvement is achieved by utilizing the different advantages of SPRT and BIET selectively, namely fast verification speed of SPRT and precise verification result of BIET (Section V-B).

The hybrid SMC technique applies SPRT and BIET selectively, because significance of verification speed and that of verification precision vary depending on a probability $p$ for $\mathcal{M}$ to satisfy a requirement property $\phi$. Suppose that if $p$ is distant from $\theta$ (e.g., $|\theta-p| \geq 0.1$ ), precision may not be very important, because small error (e.g. +0.01 or -0.01 ) in an estimated probability does not affect an accept/reject decision on $H_{0}$. In this case, the hybrid technique applies SPRT for fast verification without much concern for precision. If $p$ is close to $\theta$, however, precision becomes important, because a small error (e.g. +0.01 or -0.01) may affect an accept/reject decision on $H_{0}$ easily. In this case, the hybrid technique applies BIET for precise verification result.

Since we are targeting safety critical systems where PBLTL requirements often have $\theta$ values close to 1 (e.g., 0.99 or 0.999) for high reliability, the hybrid SMC technique can apply SPRT for relatively low $\theta_{S P R T}$ values first (e.g., 0.5, 0.75 , etc.) in hope to conclude a 'reject' decision fast with little concern for precision (a case where $p$ is distant from 
$\theta$ ). If SPRT concludes 'accept' decisions for the relatively low $\theta_{S P R T}$ s (i.e., a case where $p$ is close to $\theta$ ), the hybrid SMC algorithm applies BIET for precise verification result. Therefore, the hybrid SMC technique can produce a final verification result (i.e., accept/reject of $H_{0}$ ) fast and precisely.

Although precise verification result is of the highest priority for SMC, we cannot ignore the time cost. Since the available project time in industry is always limited, the efficiency of verification techniques is of important concern, too. For example, ISO-26262 [7] requires that the reliability of the safety critical system components should be higher than $99.999 \%$ level. To obtain such high reliability through SMC, the time cost of SMC will be significantly large (it can take several days to several weeks). Therefore, verification speed is also a critical issue as well as precision and our hybrid SMC technique can be useful for practical application of SMC techniques to improve the reliability of safety critical systems.

\section{B. Independence between Complexity of Target System and SMC Cost}

We found that the complexity of a target system does not affect the cost of the hybrid SMC technique. For example, although FFCS is more complex than the other systems (e.g., program effort $E$ of FFCS is 25500.0, while those of ATCS and ABS are 7410.9 and 3460.1 respectively (Table I)), for similar estimated probability $\hat{p}$ with the same precision parameters, a number of sample execution paths $n$ for FFCS is similar to those for ATCS and ABS. ${ }^{9}$ For the five experiments with $\alpha=\beta=0.1$ in Tables IV-VI whose $\hat{p}>0.99$, the numbers of execution paths $n$ s for these experiments are similar.

- ATCS with $\lambda=0.01$ or $0.02: \hat{p}=0.9956$ and $n=1710$

- ABS with $\lambda=0.001: \hat{p}=0.9953$ and $n=1814$

- FFCS with the sensor fault rates $(30,30,30): \hat{p}=0.9944$ and $n=2121$

- FFCS with the sensor fault rates $(20,10,20): \hat{p}=0.9920$ and $n=3180$

As shown above, although the complexities of ATCS, ABS, and FFCS are different, the cost of the hybrid SMC technique for these target systems does not change much for similar $\hat{p}$ (i.e., 0.9920-0.9956). A slightly increasing number of $n$ from 1710 to 3180 for decreasing $\hat{p}$ from 0.9956 to 0.9920 is due to the characteristics of BIET; BIET requires more sample paths as $\hat{p}$ decreases from 1 (Section II-D). Therefore, we can expect that SMC techniques can be applied to large complex safety critical systems to assure their reliability.

\section{CONCLUSION AND FUtURE WORK}

We have developed a new hybrid SMC technique which integrates SPRT and BIET. By applying this new hybrid technique to three safety critical systems in the automobile domain (i.e., ATCS, ABS, and FFCS), we have demonstrated that the hybrid SMC technique achieves precise verification results fast compared to a single SMC technique - SPRT or BIET. In our experiment, our hybrid SMC technique was up to $20 \%$ faster than BIET.

\footnotetext{
${ }^{9}$ For different target systems, we should use $n$ as a measure of the SMC cost, not time, since time varies depending on the execution time of a target system.
}

As future work, we will collaborate with Hyundai motor company to apply the hybrid SMC technique to real control components of automobiles. We believe that the hybrid technique can provide more scientific assurance about the reliability of components than conventional testing techniques. In addition, we plan to use this hybrid technique in a process to obtain an ISO-26262 certificate.

\section{ACKNOWLEDGMENTS}

This work was supported by the ERC of Excellence Program of Korea MEST/NRF of Korea) (Grant 2012-0000473) and the IT R\&D program of MKE/KEIT [10041752, Research and Development of Dual Operating System Architecture with High-Reliable RTOS and High-Performance OS].

\section{REFERENCES}

[1] D. Antic, V. Nikolic, and D. Mitic. Sliding mode control of anti-lock braking system: An overview. Automatic Control and Robotics, 9(1):4158, 2010.

[2] L. Cheung, R. Roshandel, N. Medvidovic, and L. Golubchik. Early prediction of software component reliability. In ICSE, 2008.

[3] E. Clarke, A. Biere, R. Raimi, and Y. Zhu. Bounded model checking using satisfiability solving. Formal Methods System Design (FMSD), 19(1):7-34, 2001

[4] E.M. Clarke and P. Zuliani. Statistical model checking for cyber-physical systems. In ATVA, 2011.

[5] M.H. Halstead. Elements of Software Science. Elsevier Science Ltd, 1977.

[6] T. Herault, R. Lassaigne, F. Magniette, and S. Peyronnet. Approximate probabilistic model checking. In VMCAI, 2004.

[7] International Organization for Standardization (ISO). ISO 26262: Road vehicles - functional safety, 2011. http://www.iso.org/iso/catalogue_ detail?csnumber $=43464$.

[8] S.K. Jha, E.M. Clarke, C.J. Langmead, A. Legay, A. Platzer, and P. Zuliani. A bayesian approach to model checking biological systems. In $C M S B, 2009$.

[9] Y. Kim, M. Kim, and T. Kim. Statistical model checking for safety critical hybrid systems: An empirical evaluation. In HVC, 2012.

[10] C.W. Kirhwood. System dynamics methods: A quick introduction. Technical report, 1998. Arizona State University.

[11] M. Kwiatkowska, G. Norman, and D. Parker. Prism 4.0: Verification of probabilistic real-time systems. In $C A V, 2011$.

[12] J. Lauber, T.M. Guerra, and M. Dambrine. Air-fuel ratio control in a gasoline engine. International Journal of Systems Science (IJSySc), 42(2):277-286, 2011

[13] G. Li and J. Hu. Modeling and analysis of shift schedule for automatic transmission vehicle based on fuzzy neural network. In WCICA, 2010.

[14] M.R. Lyu. Software reliability engineering: A roadmap. In Workshop on the Future of Software Engineering (FOSE), 2007.

[15] X. Teng, H. Pham, and D. R. Jeske. Reliability modeling of hardware and software interactions, and its applications. IEEE Transactions on Software Engineering (TSE), 55, 2006.

[16] A. Wald. Sequential tests of statistical hypotheses. Annals of Mathematical Statistics, 16(2):117-186, 1945.

[17] S. Yi, J. Heo, Y. Cho, and J. Hong. Adaptive mobile checkpointing facility for wireless sensor networks. In ICCSA, 2006.

[18] J. Yoo, E. Jee, and S. Cha. Formal modeling and verification of safetycritical software. IEEE Software, 26(3):42-49, 2009.

[19] H.L.S. Younes. Verification and Planning for Stochastic Processes with Asynchronous Events. PhD thesis, CMU, Jan. 2005.

[20] H.L.S. Younes, M. Kwiatkowska, G. Norman, and D. Parker. Numerical vs. statistical probabilistic model checking. Software Tools for Technology Transfer (STTT), 8(3):216-228, 2006.

[21] H.L.S. Younes and D.J. Musliner. Probabilistic plan verification through acceptance sampling. In AIPS Workshop on Planning via Model Checking, 2002.

[22] H.L.S. Younes and R.G. Simmons. Statistical probabilistic model checking with a focus on time-bounded properties. Journal Information and Computation (JIC), 204(9):1368-1409, 2006.

[23] P. Zuliani, A. Platzer, and E.M. Clarke. Bayesian statistical model checking with application to stateflow/simulink verification. In HSCC, 2010. 